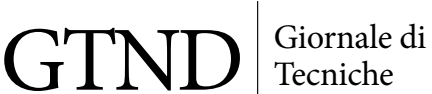 Nefrologiche e

\section{Sono una roccia per volontà mia e non per natura!}

\author{
La voce dei pazienti
}

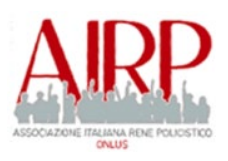

Per la prima volta in 36 anni oggi sono un po' stanca di essere quella forte sempre perché la natura mi ha fatta così, di convincermi che è merito del mio carattere e della mia soglia del dolore alta se combatto una malattia da 20 anni col sorriso... allora oggi voglio darmi il giusto valore perché forse me lo merito. Io non sono forte per natura, nessuno lo è, ho paura come tutti, sento il dolore come tutti. Sono forte perché amo chi mi sta vicino e non voglio vederli in ansia per me, e perché il mio sguardo è abbastanza lungo ed il mio cuore abbastanza grande da vedere prima chi sta peggio di chi sta meglio. La mia soglia del dolore forse è un po" altina, lo ammetto, ma le coliche renali, le crisi ipertensive, la tachicardia, i crampi, il mal di testa, il dolore cronico renale causato dalle cisti vi posso garantire che sono universali. Io combatto la malattia perché voglio farlo e non perché Nostro Signore mi ha fatta migliore degli altri o immune al dolore; lui mi ha fatta perfetta come tutti i suoi figli, non fa distinzioni lui.

Sono circa 20 anni che giro ospedali, ho fatto i peggior esami e a volte ho sentito diagnosi strampalate, altre volte
Giornale di Tecniche Nefrologiche e Dialitiche 2018, Vol. 30(3-4) 223

(C) The Author(s) 2019

Article reuse guidelines:

sagepub.com/journals-permissions

DOI: $10.1177 / 0394936218823552$

journals.sagepub.com/home/gtn

\section{(S)AGE}

non ho proprio più sentito il dottore. Mi è anche capitato di cercare di convincere i dottori che il mio fosse solamente un problema di testa, quando al mondo generalmente siamo noi pazienti a convincere il dottore del contrario, ed oggi dopo 20 anni ho trovato solo metà della diagnosi, perché l'altra metà ancora la stanno studiando geneticamente, ma io sorrido perché voglio farlo e perché sono tanto brava da saperlo fare! Non è incoscienza, non è star meno male degli altri, è essere vivi per volontà propria e non degli altri, è avere fiducia nella propria capacità di sopportazione, è essere coscienti del fatto che la vita è il dono più prezioso e non si può perdere tempo a lamentarsi!

Quindi oggi voglio essere sincera, ho dolori lancinanti per un $5 \%$ della giornata, mediamente forti per un $40 \%$, sopportabili un $20 \%$, lievi un $30 \%$ e appena percepibili un $5 \%$... ecco questa è la mia giornata, quella che da fuori non si vede, ma che da dentro riesco a dominare perché sono una roccia per volontà mia e non per natura!

Brava Federica!!! 